\title{
Eveningness and Seasonality are Associated with the Bipolar Disorder Vulnerability Trait
}

\author{
Ben Bullock • Janelle Corlass-Brown • Greg Murray
}

Published online: 5 March 2014

(C) Springer Science+Business Media New York 2014

\begin{abstract}
Trait theories of vulnerability to bipolar disorder (BD) are increasingly common in the literature, yet poorly understood. The aim of the current study was to complement existing knowledge of trait theories by investigating two biological rhythm features often associated with BD eveningness and seasonality - in a sample assessed as vulnerable to the disorder. Two hundred and thirteen participants completed an online survey consisting of the General Behavior Inventory, Seasonal Pattern Assessment Questionnaire, and Morningness-Eveningness Questionnaire. Hierarchical regressions controlling for sex and age showed that greater levels of seasonality and a tendency towards an eveningness chronotype were weak, but significant predictors of the BD vulnerability trait. When the traits of vulnerability to depression and mania were investigated separately, seasonality and eveningness were significant predictors of the former, but only seasonality was a significant predictor of the latter. The Autumn/Winter pattern of seasonality was a weak predictor of trait vulnerability to mania but not depression. The current findings advance understanding of the BD vulnerability trait, and may have consequences for the behavioural management of those who are considered to be 'at risk' of the disorder.
\end{abstract}

Keywords Bipolar disorder · Morningness · Eveningness · Seasonality $\cdot$ Vulnerability

\footnotetext{
B. Bullock $(\bowtie) \cdot \mathrm{G}$. Murray

Department of Psychological Sciences and Statistics, Swinburne University of Technology, John Street, Hawthorn, Victoria, Australia 3122

e-mail: bbullock@swin.edu.au

J. Corlass-Brown

School of Psychology (St. Patrick's), Australian Catholic University, Locked Bag 4115, Fitzroy MDC 3065, Victoria, Australia
}

As defined in DSM-5 (APA 2013), bipolar disorder (BD) is a chronic and potentially disabling mental disorder characterised by recurrent Manic, Hypomanic, and Depressive episodes. Clinical diagnosis of $\mathrm{BD}$ requires the presence of at least one Manic (BDI) or Hypomanic plus Major Depressive Episode (BDII). The model upon which diagnosis of $\mathrm{BD}$ is based is categorical, with patients classified according to whether or not they meet diagnostic criteria.

In contrast to the categorical conceptualisation that dominates classification, the quantitative trait approach to describing BD discards any notion of category and instead describes the disorder as the clinical expression of measurable traits that are distributed throughout the population (Kelsoe 2003; Frantom et al. 2008). Underpinning this approach is the argument that sets of genes and gene interactions generate neurobehavioural traits that predispose to a range of disorder phenotypes. External factors, such as psychological and environmental stressors exacerbate the trait diathesis and create the necessary conditions for expression of these phenotypes.

Studies have shown that not just BD, but a variety of mood disorders occur at higher rates in families of $\mathrm{BD}$ probands (Gershon et al. 1982; Camp et al. 2005), supportive of a broad genetic diathesis to the disorder. BD-related temperaments, shown to aggregate in the non-ill members of these families (Savitz et al. 2008; Evans et al. 2005), again suggest that risk for BD may be subject to variation along a series of vulnerability dimensions. The dimensional perspective is not unique to BD: Haslam et al. (2012) showed in a comprehensive review of the clinical literature that taxonic (categorical) models of psychological disorder were not suitable for accurate characterisation of many clinical phenomena, including anxiety, eating, and externalising disorders (e.g., aggression, psychopathy), as well as the mood disorders. Haslam et al. concluded that, on the basis of their review of taxometric research "most psychological variation is dimensional" (p. 913). 
While there is growing consensus that many psychological disorders are characteristically dimensional, the specific traits thought to underpin vulnerability to these disorders are currently unclear. In the case of $\mathrm{BD}$, a common way to conceptualise these traits is via the dopaminergic reward system model of Depue and Iacono (1989) in which vulnerability to $\mathrm{BD}$ is quantified along two separable but correlated dimensions of mania and depression. Under this model, the difference between clinical and nonclinical experience of mania and depression is merely one of arbitrary symptom quantity, and not the objective quality of symptom manifestation. The correlated two dimensional model is supported by evidence from both genetic (McGuffin et al. 2003) and psychological research (Carver and Johnson 2009; Kelly et al. 2012) in both clinical and nonclinical samples.

A focus of recent research has been the investigation of features associated with the poorly understood $\mathrm{BD}$ vulnerability trait. One area of investigation that has proven particularly fruitful is the circadian features associated with the trait. Dysregulation of the circadian system has been shown to be of etiological significance in vulnerability to $\mathrm{BD}$, being demonstrated at molecular, physiological, and behavioural levels in clinical and non-clinical samples (see Murray and Harvey 2010). It has also been shown that social rhythmicity (a downstream expression of the endogenous circadian pacemaker) is significantly reduced in non-clinical participants assessed to be at higher risk of $\mathrm{BD}$ compared to a nonclinical, lower risk group (Bullock et al. 2011). In another study, higher risk participants demonstrated greater instability in their 24-hour activity patterns than their lower risk counterparts (Bullock and Murray 2013). Risk for BD was defined in these studies as the combined level of dimensional vulnerability to both mania and depression, and measured using the General Behavior Inventory (GBI; Depue et al. 1989). Outcomes from both of these studies are consistent with wellestablished findings from previous investigations showing disruption of downstream circadian processes in BD samples (e.g., Ashman et al. 1999; Shen et al. 2008; Boland et al. 2012). The current investigation focuses on two further biological rhythm features commonly associated with BD eveningness and seasonality.

\section{Eveningness and Vulnerability to BD}

Phase delay of biological rhythm processes is a common trait manifestation of circadian system dysregulation in people with BD. Later peaks in the 24-hour rhythms of core body temperature (e.g., Daimon et al. 1992; Souetre et al. 1988) and melatonin onset (e.g., Nurnberger et al. 2000; Nathan et al. 1999) have been demonstrated. In addition to these experimental findings, chronobiological treatment of BD depressive states can involve phase advance of the sleep/wake cycle and is effective in many cases (Dallaspezia and Benedetti 2011). Such findings are consistent with a realignment of the sleep/ wake cycle and the endogenous circadian rhythm.

Greater prevalence of the eveningness chronotype amongst BD samples provides further supportive evidence of phase delayed circadian processes in this population. The eveningness chronotype is associated with a preference for evening, as opposed to morning, activity. Those with a preference for eveningness tend to go to bed and wake up later, with performance and awareness levels peaking in the latter part of the day. Wood et al. (2009) showed that a group of euthymic outpatients with BD (either I or II) reported greater eveningness preference than a group of non-clinical controls. Mansour et al. (2005) reported similar findings in a sample of euthymic patients with BD-I, as did Ahn et al. (2008) in a sample of Korean patients with BD-I, supporting the prevalence of the later chronotype in clinically stable, euthymic samples.

Differences in circadian phase and circadian preference have also been demonstrated in non-clinical samples at putative risk of BD. Ankers and Jones (2009) showed that participants assessed to be at high behavioural risk of BD reported significantly later bed times than a group of participants assessed to be at low behavioural risk. Behavioural risk in this study was defined using scores on the Hypomanic Personality Scale (HPS; Eckblad and Chapman 1986), a validated scale which measures the $\mathrm{BD}$ vulnerability trait on a single hypomania-related dimension. Ottoni et al. (2011) also showed that the cyclothymic temperament (strongly linked to BD-II; Koukopoulos et al. 2006) was associated with later sleep and wake times. Closely related to these findings was the association demonstrated between greater eveningness and minor psychiatric disorders amongst a sample of University students (de Medeiros Schneider et al. 2011). Hakkarainen et al. (2003) showed that healthy twin siblings did not differ in eveningness scores from their BD-I co-twin, although neither group reported scores within the eveningness range as defined by Horne and Ostberg (1976). Overall however, it would appear that an orientation towards eveningness is associated with vulnerability to $\mathrm{BD}$, and this is consistent with a possible phase delay in biological rhythms that has been observed in people with BD.

\section{Seasonality and Vulnerability to BD}

Seasonal changes in mood, behaviour, and social activity are inextricably linked to dysregulation of the circadian system via serotonergic pathways (Ciarleglio et al. 2011) and, as such, are relatively common in people with BD. Shand et al. (2011), for example, reported that $27 \%$ of patients with $\mathrm{BD}$ fulfilled criteria for Seasonal Affective Disorder (SAD), as measured with the Seasonal Pattern Assessment Questionnaire (SPAQ; 
Rosenthal et al. 1984), a prevalence rate approximately 3-4 times higher than a comparison sample of patients in primary care. Similar rates of SAD prevalence amongst BD populations have been reported elsewhere (Schaffer et al. 2003; Simonsen et al. 2011; Goikolea et al. 2007), although a recent prospective study failed to find evidence of a seasonal pattern in episodes of either mania or depression (Murray et al. 2011). Investigations of hospital admissions for depression and mania typically report an increase in depressive presentations in Autumn/Winter and an increase in manic presentations in Spring/Summer (Lee et al. 2007). Again, however, this pattern failed to replicate in the prospective investigation of manic and depressive symptoms by Murray et al. (2011)

Investigations of $\mathrm{SAD}$, in both its clinical and non-clinical forms ('seasonality'), amongst people at risk of BD are scarce. A study comparing same-sex twins, one of whom had a confirmed diagnosis of BD-I, showed significantly lower seasonal fluctuation in mood and sleep in the healthy twin (Hakkarainen et al. 2003). The healthy twin group in this study also had significantly lower Global Seasonality Scores (GSS) on the SPAQ than their twin siblings with BD-I. Mean GSS for the healthy twin in this study was 5.20, which is consistent with mean GSS for normative samples in other studies (e.g., Murray 2003). Another study showed that symptoms of depression and anxiety in a large community sample were positively correlated with GSS scores (Oyane et al. 2008), however hypomanic symptomatology was not assessed in this study. Parslow, Jorm, Butterworth, Jacomb, and Rogers (2004) also reported a positive association between depression, anxiety, and seasonality. In addition, their seasonal group reported significantly higher levels of Neuroticism, a trait often associated with mood disorders in general, than their non-seasonal group. There were no group differences however, on Extraversion, BAS-drive, and BAS-reward responsiveness, traits that are commonly associated more specifically with BD (see, for example, Johnson et al. 2012). Evidence of seasonality as an associated factor in trait vulnerability to BD is therefore mixed. However, very few studies have investigated seasonality in samples assessed to have greater trait vulnerability to $\mathrm{BD}$ specifically.

The aim of the current study was to investigate two readily measured biological rhythm features associated with BD (seasonality and eveningness) in a non-clinical sample quantified by vulnerability to $\mathrm{BD}$. Consistent with trait vulnerability models of $\mathrm{BD}$, it was hypothesised that a positive association between vulnerability to BD and eveningness would be found. The mixed findings on trait vulnerability to BD and seasonality preclude a definitive hypothesis regarding their relationship. Nevertheless, the potential for such a relationship on theoretical grounds means that this relationship was also investigated. Eveningness and seasonality as predictors of separate Depression and Mania traits were also investigated. While BD follows a predominantly depressive course (Judd et al. 2003), the defining feature of the disorder is mania (e.g., Schweitzer et al. 2005), and this actuality formed the basis of our predictions. Thus, it was predicted that the Mania trait would be more strongly associated with eveningness and seasonality than the Depression trait (see also Bullock and Murray 2013).

\section{Method}

Participants

The sample consisted of 213 participants (178 women and 35 men) recruited from the student body of a university in Melbourne, Australia. Participants were aged between 18 and 30 years of age. The restricted age range served two purposes. First, age is a known moderator of chronotype (e.g., Carrier et al. 1997). Second, a university student-based sample lends suitable characterisation of the trait vulnerability construct as the typical age range of such a sample provides sufficient examples of subsyndromal BD-related disturbance, as well as a broad range of mood-related "phenotypic intensities" (Depue et al. 1989; p. 118). Four participants (all women) reported being previously diagnosed with $\mathrm{BD}$ and were subsequently excluded from further analysis. Excluding participants with a history of BD sharpens the focus of the research on those who are potentially at risk of $\mathrm{BD}$, but who have not had a hypomanic or manic episode. Interpretation of outcomes is simpler because important relationships cannot be attributed to sequelae of the disorder.

\section{Materials \& Procedures}

\section{General Behavior Inventory}

The BD vulnerability trait was measured using the General Behavior Inventory (GBI; Depue et al. 1989), a 73-item selfreport inventory of BD-related experiences that incorporates elements of intensity, duration, and frequency of symptomatology. Total GBI score is the sum of scores on two subscales measuring Depression- and Mania-proneness. The Depression scale of the GBI consists of 46 items and the Mania scale consists of 27 items. GBI Total scores range from 0 to 219 with higher scores used to indicate greater trait vulnerability to BD.

The GBI has proved to be psychometrically sound and has demonstrated high specificity and adequate sensitivity to identify both syndromal and subsyndromal affective conditions in both clinical and non-clinical populations (Depue and Klein 1988; Reichart et al. 2004; Findling et al. 2002). The GBI has also been used successfully as a screening tool for bipolar spectrum conditions in the large longitudinal investigation run through Temple University and University of Wisconsin 
(Alloy et al. 2009). The GBI was shown to have good predictive validity of bipolar spectrum conditions in this large study.

\section{Seasonal Pattern Assessment Questionnaire}

Seasonality was measured with the Seasonal Pattern Assessment Questionnaire (SPAQ; Rosenthal et al. 1984). The instrument measures the degree and pattern of seasonal change in mood and behaviour as well as the subjective level of distress these changes cause. The Global Seasonality score (GSS) is calculated as the total of the responses on six change items and is commonly used to describe the extent of seasonality, with higher scores indicating greater seasonal variation, or higher seasonality. Using a 5-point Likert scale from no change $(=0)$ to extremely marked change $(=4)$ an example change item is "the degree to which sleep length changes with the seasons". Total scores range from 0 to 24 . The SPAQ also allows respondents to record the months of the year in which they 'feel worst', if any. In the current study, an Autumn/Winter pattern was indicated if the respondent reported feeling worst in any of the months from April to August, while a Spring/Summer pattern was indicated if any of the months from September to February was reported. ${ }^{1}$ The psychometric properties of the SPAQ are sound. Test-retest reliability was 0.76 and internal consistency was 0.81 in a study of college students (Young et al. 2003). The SPAQ has also been shown to adequately discriminate between seasonal and non-seasonal groups, such as those with and without SAD (Mersch et al. 2004).

\section{Morningness-Eveningness Questionnaire}

Eveningness was assessed using the 19-item MorningnessEveningness Questionnaire (MEQ; Horne and Ostberg 1976). Scores range from 16 to 86, with lower scores indicating greater eveningness (see Natale and Cicogna 2002). The MEQ items require participants to indicate their preference for morning or evening activity in a range of circumstances. For example: "Assuming adequate environmental conditions, how easy do you find getting up in the mornings?" A 4-point Likert scale is used for most items, with a further five items requiring participants to mark responses along an hourly time line. Good internal consistency has been reported for the MEQ (Roberts and Kyllonen 1999; Smith et al. 1989). Validation with external criteria has also been demonstrated (Smith et al. 1989; Horne and Ostberg 1976). For the purposes of the current study only, MEQ scores were reverse-coded so that higher scores indicated an orientation towards an eveningness chronotype (cf. Hakkarainen et al. 2003)

Participants completed all scales anonymously and online. Ethical approval for study procedures was gained from the local university Human Research Ethics Committee.

\footnotetext{
${ }^{1}$ These patterns refer to data collected in the southern hemisphere only.
}

Participants' informed consent was implied from submission of the questionnaire.

Design \& Analysis

Hierarchical regressions were used for hypothesis testing. Sex was entered at Step 1 for all analyses due to the disproportionate number of women in the sample. There is also some evidence to suggest that sex may moderate chronotype (e.g., Adan and Natale 2002) and seasonality (Tonetti et al. 2007) so this variable was statistically controlled by entering it at Step 1 . The MEQ and GSS predictors were entered together at Step 2.

The distribution of scores on the MEQ and GSS scales was normal. Not unexpectedly, the distributions of scores on the GBI-Depression and GBI-Mania scales were positively skewed. Furthermore, the scatterplot of standardised residuals for both scales revealed significant heteroscedasticity. A square root transformation of data on both scales normalised distributions and homoscedasticity. The transformed variable in each case was used for hypothesis testing. ${ }^{2}$ All other assumptions were met.

\section{Results}

\section{Descriptive Data}

Correlations, descriptive statistics, and scale reliabilities are presented in Table 1. Mean scores for all scales are consistent with those of previous studies using similar populations (Murray et al. 2007; de Medeiros Schneider et al. 2011; Young et al. 2003) and reliability was good to excellent for all scales. The magnitude and direction of correlations between the variables were largely as expected based on theory and previous research (e.g., Shand et al. 2011; Wood et al. 2009).

\section{Hypothesis Testing}

For the first analysis, GSS and MEQ scores were used to predict GBI-Total. Two multivariate outliers were removed prior to analysis. Sex was entered at Step 1 as a covariate followed by GSS and MEQ at Step 2. The model at Step 1 was not significant; $R^{2}=0.00, F(1,205)=0.001, p=0.98$. With the addition of GSS and MEQ at Step 2 the model was significant; $R^{2} \Delta=0.10, F(2,203)=11.12, p<0.001$. Table 2 shows that both GSS and MEQ were significant predictors of GBI-Total, with the former predictor explaining more unique variance than the latter.

\footnotetext{
${ }^{2}$ The untransformed data was used for the logistic regression analysis predicting the pattern of seasonality from GBI scores due to the less restrictive distributional assumptions associated with this test.
} 
Table 1 Correlations, descriptive data, and Cronbach's Alpha for the GBI, MEQ, and GSS

\begin{tabular}{llllll}
\hline & 1 & 2 & 3 & 4 & 5 \\
& GBI-Depression & GBI-Mania & GBI-Total & MEQ & GSS \\
\hline 1 & - & $0.79^{* *}$ & $0.98^{* *}$ & $0.18^{*}$ & $0.21^{*}$ \\
2 & & - & $0.90^{* *}$ & 0.14 & $0.26^{*}$ \\
3 & & & - & $0.18^{*}$ & $0.24^{*}$ \\
4 & & & & - & 0.04 \\
5 & & & & & - \\
$M$ & 31.31 & 17.45 & 48.76 & 49.58 & 9.29 \\
$S D$ & 25.34 & 12.37 & 35.88 & 8.59 & 4.87 \\
range & $0-111$ & $0-70$ & $0-156$ & $27-71$ & $0-23$ \\
Reliability & 0.97 & 0.94 & 0.98 & 0.77 & 0.84 \\
\hline
\end{tabular}

GBI General Behavior Inventory, $M E Q$ Morningness Eveningness Questionnaire, GSS Global Seasonality Score

$N=209$

$* * p<0.001$

$* p<0.01$

For the second and third analyses, the relationship between the predictors and GBI score was investigated separately for the Depression and Mania scales. Sex was again entered at Step 1 followed by the GSS and MEQ predictors at Step 2. Two multivariate outliers were removed from each analysis, respectively. The regression table for predicting GBI-Depression is presented in Table 3 and the regression table for predicting GBIMania is presented in Table 4.

For the GBI-Depression analysis the model at Step 1 was not significant; $R^{2}=0.00, F(1,205)=0.22, p=0.64$. With the addition of GSS and MEQ at Step 2 the model was significant; $R^{2} \Delta=0.09, F(2,203)=9.50, p<0.001$. Both GSS and MEQ were significant predictors of GBI-Depression, with the former predictor explaining more unique variance than the latter.

Table 2 Predicting GBI-total from GSS and MEQ: hierarchical regression

\begin{tabular}{ccclc}
\hline Variable & $B$ & $S E B$ & $\beta$ & $s r^{2}$ \\
\hline $\begin{array}{rlll}\text { Step 1 } \\
\text { Sex }\end{array}$ & 0.01 & 0.50 & 0.00 & 0.00 \\
Step 2 & & & & \\
Sex & 0.25 & 0.50 & 0.04 & 0.00 \\
GSS & 0.15 & 0.04 & $0.27^{* *}$ & 0.07 \\
MEQ & 0.05 & 0.02 & $0.17^{*}$ & 0.03 \\
\hline
\end{tabular}

MEQ Morningness Eveningness Questionnaire, GSS Global Seasonality Score

$N=207$

$* * p<0.001$

$* p<0.05$
Table 3 Predicting GBI-depression from GSS and MEQ: hierarchical regression

\begin{tabular}{lllll}
\hline Variable & $B$ & $S E B$ & $\beta$ & $s r^{2}$ \\
\hline $\begin{array}{l}\text { Step 1 } \\
\text { Sex }\end{array}$ & -0.20 & 0.44 & -0.03 & 0.00 \\
Step 2 & & & & \\
Sex & -0.05 & 0.44 & -0.01 & 0.00 \\
GSS & 0.12 & 0.03 & $0.24^{*}$ & 0.05 \\
MEQ & 0.05 & 0.02 & $0.18^{*}$ & 0.03 \\
\hline $\begin{array}{l}M E Q \text { Morningness Eveningness Questionnaire, GSS Global Seasonality } \\
\text { Score }\end{array}$ & & & \\
$N=207$ & & & \\
$* p<0.01$ & & &
\end{tabular}

For the GBI-Mania analysis the model at Step 1 was not significant; $R^{2}=0.01, F(1,205)=1.07, p=0.30$. With the addition of GSS and MEQ at Step 2 the model was significant; $R^{2} \Delta=0.10, F(2,203)=11.41, p<0.001$. GSS, but not MEQ, was a significant predictor of GBI-Mania, explaining $8.8 \%$ unique variance.

Separating the prediction of GBI into the Depression and Mania subscales showed that GSS was a stronger predictor of GBI-Mania than GBI-Depression. The relationship between GSS and GBI-Mania was positive, indicating that greater levels of seasonality were associated with higher GBI-Mania scores. Alternatively, MEQ was a stronger predictor of GBIDepression than GBI-Mania, with the positive relationship indicating that greater eveningness was associated with higher GBI-Depression scores. ${ }^{3}$

A follow-up analysis investigated the predictors of the Autumn/Winter seasonality type. A hierarchical logistic regression with Autumn/Winter type the outcome variable and GBI-Depression and GBI-Hypomania the predictors was conducted. Again, sex was a covariate, and again, two multivariate outliers were removed from the analysis. Table 5 presents the outcome of the logistic regression.

Table 5 shows that Sex and GBI-Mania, but not GBIDepression, were significant predictors of an Autumn/Winter pattern of seasonality in this sample. The odds ratio for the Sex predictor variable at Step 2 indicates that women were 3.56 times more likely to report an Autumn/Winter pattern than men. The odds ratio for the GBI-Mania variable at Step 2 indicates that for higher GBI-Mania scores there was a slightly greater likelihood of an Autumn/Winter pattern of seasonality.

The majority of participants (70.5\%) reported that changes in mood and behaviour with the seasons were not a problem for them. For those that did report it as a problem, the modal level of severity was at the lowest end of the scale ('Mild'=

\footnotetext{
${ }^{3}$ Recall that MEQ scores were reverse-scored so that orientation towards an eveningness chronotype was reflected in higher MEQ scores.
} 
Table 4 Predicting GBI-mania from GSS and MEQ: hierarchical regression

\begin{tabular}{cllll}
\hline Variable & $B$ & $S E B$ & $\beta$ & $s r^{2}$ \\
\hline Step 1 & & & & \\
Sex & 0.31 & 0.30 & 0.07 & 0.01 \\
Step 2 & & & & \\
Sex & 0.52 & 0.30 & 0.12 & 0.01 \\
GSS & 0.10 & 0.02 & $0.30^{*}$ & 0.09 \\
MEQ & 0.02 & 0.01 & 0.12 & 0.01 \\
\hline
\end{tabular}

$M E Q$ Morningness Eveningness Questionnaire, GSS Global Seasonality Score

$N=207$

${ }^{*} p<0.001$

$43.2 \%)$, followed by 'Moderate' (28.4\%), and 'Marked' $(23.0 \%)$. Only $5.4 \%$ of the sample rated the changes as 'Severe' or 'Disabling'.

\section{Discussion}

This study investigated two commonly recognised biological rhythm features associated with BD in a group of participants assessed for trait vulnerability to the disorder. It was hypothesised that there would be a positive association between vulnerability to BD and the eveningness chronotype, and that vulnerability to mania specifically would be more strongly related to this chronotype than vulnerability to depression. The relationship between trait vulnerability to $\mathrm{BD}$ and seasonality of mood and behaviour was also investigated, although specific hypotheses were not formulated due to mixed findings in previous research.

The positive relationship between MEQ scores and GBITotal scores supported the first component of the first hypothesis. Greater trait vulnerability to BD was associated with an orientation towards the eveningness chronotype, albeit at a small effect size. The second component of the first hypothesis was not supported. Indeed the opposite was found, with MEQ scores explaining more unique variance in GBIDepression than in GBI-Mania. Again, effect sizes were small.

The findings regarding the relationship between trait vulnerability to BD and an eveningness chronotype are consistent with previous findings reported by Wood et al. (2009), Mansour et al. (2005), and Ahn et al. (2008) in their clinical BD samples. All studies showed that vulnerability to BD was associated with an eveningness chronotype. In the current study, the effect size of this relationship was small compared to the medium effect size of relationships reported in the clinical samples, particularly Wood et al. and Mansour et al. The smaller effect size in the current study may be attributable to the non-clinical nature of the sample. Illness progression in those diagnosed with BD may amplify circadian-related effects due to repeated allostatic states and subsequent kindling processes (see Kapczinski et al. 2008). Such effects are not apparent in non-clinical samples.

Finding a stronger relationship between depression and eveningness than between mania and eveningness was unexpected. Stronger circadian-related effects are reported in mania than depression (Bullock and Murray 2013) and a substantial body of literature supports a significant role for circadian-related effects in BD versus Major Depressive Disorder (e.g., Lamont et al. 2007). A possible explanation for this unexpected finding is that clinical states of mania are relatively uncommon compared to clinical states of depression, and this difference in symptomatic status likely extends to subsyndromal expressions of these states (Judd et al. 2003) and possibly to the traits of mania and depression as investigated in the current study. There may be limited power therefore to adequately characterise the relationship between the dimensional trait of mania and the eveningness chronotype.

Investigation of the relationship between seasonality and trait vulnerability to $\mathrm{BD}$ revealed that seasonality was a significant, positive, and weak-to-moderate predictor of trait vulnerability to BD. The current findings appear to be consistent therefore with those of Shand et al. (2011), Simonsen

Table 5 Predicting autumn/winter seasonality type from GBI-depression and GBI-mania: hierarchical logistic regression

\begin{tabular}{|c|c|c|c|c|c|c|}
\hline Variables & $B$ & $S E B$ & Wald Chi-square & $d f$ & $p$ & Odds Ratio \\
\hline \multicolumn{7}{|l|}{ Step 1} \\
\hline Sex & 1.09 & 0.38 & 8.17 & 1 & $<0.01$ & 2.97 \\
\hline \multicolumn{7}{|l|}{ Step 2} \\
\hline Sex & 1.27 & 0.41 & 9.70 & 1 & $<0.01$ & 3.56 \\
\hline GBI-Depression & -0.00 & 0.01 & 0.05 & 1 & 0.82 & 1.00 \\
\hline GBI-Mania & -0.05 & 0.02 & 4.89 & 1 & $<0.05$ & 0.95 \\
\hline Constant & 0.14 & 0.26 & 0.27 & 1 & 0.61 & 1.15 \\
\hline
\end{tabular}

The Sex predictor variable was coded as 0 for female and 1 for male. Higher scores on the GBI-Depression and GBI-Mania scales indicate greater presence of the trait. Autumn/Winter pattern of seasonality was the reference category 
et al. (2011), Goikolea et al. (2007), and Schaffer et al. (2003), in finding that greater vulnerability to $\mathrm{BD}$ is associated with greater seasonal fluctuation in mood and behaviour, but not consistent with the prospective investigation of Murray et al. (2011). The results are partially consistent with Oyane et al. (2008) and Parslow et al. (2004) in finding that seasonality is associated with vulnerability to mood disorders in general. The Autumn/Winter pattern of seasonality was associated with trait vulnerability to mania, consistent with Lee et al. (2007), but not with trait vulnerability to depression.

The most important predictor of the Autumn/Winter pattern of seasonality in the current study was participants' sex. Female participants were 3.5 times more likely to report this pattern than men. This finding is consistent with previous research in this area showing that women are more likely to report an Autumn/Winter pattern of depression than men (e.g., Suhail and Cochrane 1998). It is possible however, that this relationship in the current study is an artefact of the sample, with substantially more women participating in the study than men.

Over two-thirds of participants felt that seasonal changes in mood and behaviour were 'not a problem for them'. In contrast, $46 \%$ of the BD sample of Simonsen et al. (2011) reported that the changes were at least a moderate problem for them. In the current study, those that did report that these changes were a problem, only $5.4 \%$ reported that they were 'severe' or 'disabling', and this figure is lower than that reported for the BD sample of Simonsen et al. (9.6\%). Whether these differences in the perceived level of the problem and the severity of seasonal changes between BD samples and vulnerability trait samples are part of the progression of the $\mathrm{BD}$ illness requires further investigation using objective measures of seasonal changes in behaviour. It could however, also suggest that the interpretation of these changes is modifiable, and that cognitive-behavioural techniques may help people with BD cope with seasonal changes in mood and behaviour if they are causing problems. Harvey et al. (2005) raised a similar possibility in their investigation of subjective and objective sleep measurements in people with BD.

The study had a number of limitations. Seasonality and eveningness were measured using self-report instruments. Independent measurement of these constructs could be facilitated through actigraphy or other behavioural pattern monitors (e.g., Prociow and Crowe 2010; Bardram et al. 2012) and this would represent a more objective investigation of the seasonality and eveningness constructs. The ease with which these technologies can be applied may also encourage longitudinal investigation of the relevant constructs, which would also represent a methodological advance on the current investigation. A better distribution of demographic features is also recommended for future studies of this kind. In particular, a more even balance of males and females is suggested. Finally, structured clinical interviews of non-clinical participants would provide a more rigourous test of the absence of $\mathrm{BD}$ diagnosis (both current and historical) than the self-report method used in the current study.

The aim of the current study was to advance understanding of trait vulnerability to BD by focusing on two commonly observed circadian-related features of the disorder. Relationships emerged largely in the predicted direction in this non-clinical sample, although small effect sizes were a limiting feature. Nevertheless, the current findings add incrementally to the emerging picture of the trait vulnerability concept, specifically as it relates to $\mathrm{BD}$, and may have consequences for the behavioural management of those who are 'at risk' of mania and depression.

Conflict of Interest The authors declare that they have no conflicts of interest with respect to the authorship, conduct, or publication of this article.

Experiment Participants Ethical approval for the study was gained from the local university Human Research Ethics Committee and study procedures comply with the Australian Code for Responsible Conduct of Research. Participants' informed consent was implied from submission of the anonymous questionnaire.

\section{References}

Adan, A., \& Natale, V. (2002). Gender differences in morningnesseveningness preference. Chronobiology International, 19(4), 709720. doi:10.1081/cbi-120005390.

Ahn, Y. M., Chang, J., Joo, Y. H., Kim, S. C., Lee, K. Y., \& Kim, Y. S. (2008). Chronotype distribution in bipolar I disorder and schizophrenia in a Korean sample. Bipolar Disorders, 10(2), 271-275.

Alloy, L. B., Abramson, L. Y., Walshaw, P. D., Gerstein, R. K., Keyser, J. D., Whitehouse, W. G., et al. (2009). Behavioral Approach System (BAS)-relevant cognitive styles and bipolar spectrum disorders: concurrent and prospective associations. Journal of Abnormal Psychology, 118(3), 459-471.

Ankers, D., \& Jones, S. H. (2009). Objective assessment of circadian activity and sleep patterns in individuals at behavioral risk of hypomania. Journal of Clinical Psychology, 65(10), 1071-1086. doi:10. $1002 /$ jclp. 20608 .

APA. (2013). DSM-5. Washington, DC: Author.

Ashman, S. B., Monk, T. H., Kupfer, D. J., Clark, C. H., Myers, F. S., Frank, E., et al. (1999). Relationship between social rhythms and mood in patients with rapid cycling bipolar disorder. Psychiatry Research, 86, 1-8.

Bardram, J. E., Frost, M., Szántó, K., \& Marcu, G. The MONARCA selfassessment system - A persuasive personal monitoring system for bipolar patients. In 2nd ACM SIGHIT International Health Informatics Symposium, Miami, FL, 2012 (pp. 21-30, 2nd ACM SIGHIT International Health Informatics Symposium, IHI'12). doi: 10.1145/2110363.2110370.

Boland, E. M., Bender, R. E., Alloy, L. B., Conner, B. T., LaBelle, D. R., \& Abramson, L. Y. (2012). Life events and social rhythms in bipolar spectrum disorders: an examination of social rhythm sensitivity. Journal of Affective Disorders, 139(3), 264-272. doi:10.1016/j.jad. 2012.01.038.

Bullock, B., \& Murray, G. (2013). Reduced amplitude of the 24-hr activity rhythm: a biomarker of vulnerability to bipolar disorder? [Advance online publication]. Clinical Psychological Science. doi: $10.1177 / 2167702613490158$.

Bullock, B., Judd, F., \& Murray, G. (2011). Social rhythms and vulnerability to bipolar disorder. [Brief Report]. Journal of Affective Disorders, 135(1-3), 384-388. 
Camp, N. J., Lowry, M. R., Lynn Richards, R., Plenk, A. M., Carter, C., Hensel, C. H., et al. (2005). Genome-wide linkage analyses of extended Utah pedigrees identifies loci that influence recurrent, early-onset major depression and anxiety disorders. American Journal of Medical Genetics - Neuropsychiatric Genetics, 135 $B(1), 85-93$.

Carrier, J., Monk, T. H., Buysse, D. J., \& Kupfer, D. J. (1997). Sleep and morningness-eveningness in the "middle" years of life (20-59 y). Journal of Sleep Research, 6, 230-237.

Carver, C. S., \& Johnson, S. L. (2009). Tendencies toward mania and tendencies toward depression have distinct motivational, affective, and cognitive correlates. Cognitive Therapy and Research, 33(6), $552-569$.

Ciarleglio, C. M., Resuehr, H. E. S., \& McMahon, D. G. (2011). Interactions of the serotonin and circadian systems: nature and nurture in rhythms and blues. Neuroscience, 197, 8-16. doi:10. 1016/j.neuroscience.2011.09.036.

Daimon, K., Yamada, N., Tsujimoto, T., \& Takahashi, S. (1992). Circadian rhythm abnormalities of deep body temperature in depressive disorders. Journal of Affective Disorders, 26, 191-198.

Dallaspezia, S., \& Benedetti, F. (2011). Chronobiological therapy for mood disorders. Expert Review of Neurotherapeutics, 11(7), 961-970.

de Medeiros Schneider, M. L., Vasconcellos, D. C., Dantas, G., Levandovski, R., Caumo, W., Allebrandt, K. V., et al. (2011). Morningness-eveningness, use of stimulants, and minor psychiatric disorders among undergraduate students. International Journal of Psychology, 46(1), 18-23. doi:10.1080/00207594.2010.513414.

Depue, R. A., \& Iacono, W. G. (1989). Neurobehavioural aspects of affective disorders. Annual Review of Psychology, 40, 457-492.

Depue, R. A., \& Klein, D. N. (1988). Identification of unipolar and bipolar affective conditions in nonclinical and clinical populations by the General Behavior Inventory. In D. L. Dunner, E. S. Gershon, \& J. E. Barrett (Eds.), Relatives at risk for mental disorder (pp. 179204). New York: Raven Press, Ltd.

Depue, R. A., Krauss, S., Spoont, M. R., \& Arbisi, P. (1989). General behaviour inventory identification of unipolar and bipolar affective conditions in a nonclinical university population. Journal of Abnormal Psychology, 98(2), 117-126.

Eckblad, M., \& Chapman, L. J. (1986). Development and validation of a scale for hypomanic personality. Journal of Abnormal Psychology, 95(3), 214-222.

Evans, L., Akiskal, H. S., Keck, P. E., Jr., McElroy, S. L., Sadovnick, A. D., Remick, R. A., et al. (2005). Familiality of temperament in bipolar disorder: support for a genetic spectrum. Journal of Affective Disorders, 85(1-2), 153-168.

Findling, R. L., Youngstrom, E. A., Danielson, C. K., DelPorto-Bedoya, D., Papish-David, R., Townsend, L., et al. (2002). Clinical decisionmaking using the General Behavior Inventory in juvenile bipolarity. Bipolar Disorders, 4(1), 34-42.

Frantom, L. V., Allen, D. N., \& Cross, C. L. (2008). Neurocognitive endophenotypes for bipolar disorder. Bipolar Disorders, 10(3), 387399. doi:10.1111/j.1399-5618.2007.00529.x.

Gershon, E. S., Hamovit, J., Guroff, J. J., Dibble, E., Leckman, J. F., Sceery, W., et al. (1982). A family study of schizoaffective, bipolar I, bipolar II, unipolar, and normal control probands. Archives of General Psychiatry, 39(10), 1157-1167.

Goikolea, J. M., Colom, F., Martínez-Arán, A., Sánchez-Moreno, J., Giordano, A., Bulbena, A., et al. (2007). Clinical and prognostic implications of seasonal pattern in bipolar disorder: a 10 -year follow-up of 302 patients. Psychological Medicine, 37(11), 15951599. doi:10.1017/s0033291707000864.

Hakkarainen, R., Johansson, C., Kieseppa, T., Partonen, T., Koskenvuo, M., Kaprio, J., et al. (2003). Seasonal changes, sleep length and circadian preference among twins with bipolar disorder. $B M C$ Psychiatry, 3, 6.
Harvey, A. G., Schmidt, D. A., Scarna, A., Semler, C. N., \& Goodwin, G. M. (2005). Sleep-related functioning in euthymic patients with bipolar disorder, patients with insomnia, and subjects without sleep problems. American Journal of Psychiatry, 162(1), 50-57.

Haslam, N., Holland, E., \& Kuppens, P. (2012). Categories versus dimensions in personality and psychopathology: a quantitative review of taxometric research. Psychological Medicine, 42(5), 903-920. doi:10.1017/s0033291711001966.

Horne, J. A., \& Ostberg, O. (1976). A self-assessment questionnaire to determine morningness-eveningness in human circadian rhythms. International Journal of Chronobiology, 4, 97-110.

Johnson, S. L., Edge, M. D., Holmes, M. K., \& Carver, C. S. (2012). The behavioral activation system and mania. Annual Review of Clinical Psychology, 8, 243-267.

Judd, L. L., Akiskal, H. S., Schettler, P. J., Coryell, W., Endicott, J., Maser, J. D., et al. (2003). A prospective investigation of the natural history of the long-term weekly symptomatic status of bipolar II disorder. Archives of General Psychiatry, 60(3), 261-269. doi:10. 1001/archpsyc.60.3.261.

Kapczinski, F., Vieta, E., Andreazza, A. C., Frey, B. N., Gomes, F. A., Tramontina, J., et al. (2008). Allostatic load in bipolar disorder: implications for pathophysiology and treatment. Neuroscience and Biobehavioral Reviews, 32(4), 675-692.

Kelly, R. E., Mansell, W., Sadhnani, V., \& Wood, A. M. (2012). Positive and negative appraisals of the consequences of activated states uniquely relate to symptoms of hypomania and depression. Cognition and Emotion, 26(5), 899-906.

Kelsoe, J. R. (2003). Arguments for the genetic basis of the bipolar spectrum. Journal of Affective Disorders, 73(1-2), 183-197.

Koukopoulos, A., Sani, G., Koukopoulos, A. E., Albert, M. J., Girardi, P., \& Tatarelli, R. (2006). Endogenous and exogenous cyclicity and temperament in bipolar disorder: review, new data and hypotheses. Journal of Affective Disorders, 96(3), 165-175. doi:10.1016/j.jad. 2006.08.031.

Lamont, E. W., Legault-Coutu, D., Cermakian, N., \& Boivin, D. B. (2007). The role of circadian clock genes in mental disorders. Dialogues in Clinical Neuroscience, 9(3), 333-342.

Lee, H. C., Tsai, S. Y., \& Lin, H. C. (2007). Seasonal variations in bipolar disorder admissions and the association with climate: a populationbased study. Journal of Affective Disorders, 97(1-3), 61-69.

Mansour, H. A., Wood, J., Chowdari, K. V., Dayal, M., Thase, M. E., Kupfer, D. J., et al. (2005). Circadian phase variation in bipolar I disorder. Chronobiology International, 22(3), 571-584.

McGuffin, P., Rijsdijk, F., Andrew, M., Sham, P., Katz, R., \& Cardno, A. (2003). The heritability of bipolar affective disorder and the genetic relationship to unipolar depression. Archives of General Psychiatry, $60,497-502$

Mersch, P. P. A., Vastenburg, N. C., Meesters, Y., Bouhuys, A. L., Beersma, D. G. M., Van Den Hoofdakker, R. H., et al. (2004). The reliability and validity of the Seasonal Pattern Assessment Questionnaire: a comparison between patient groups. Journal of Affective Disorders, 80(2-3), 209-219. doi:10.1016/s01650327(03)00114-9.

Murray, G. (2003). The Seasonal Pattern Assessment Questionnaire as a measure of mood seasonality: a prospective validation study. Psychiatry Research, 120(1), 53-59.

Murray, G., \& Harvey, A. (2010). Circadian rhythms and sleep in bipolar disorder. Bipolar Disorders, 12(5), 459-472. doi:10.1111/j.13995618.2010.00843.x.

Murray, G., Goldstone, E., \& Cunningham, E. (2007). Personality and the predisposition(s) to bipolar disorder: heuristic benefits of a twodimensional model. Bipolar Disorders, 9(5), 453-461. doi:10. 1111/j.1399-5618.2007.00456.x.

Murray, G., Lam, R. W., Beaulieu, S., Sharma, V., Cervantes, P., Parikh, S. V., et al. (2011). Do symptoms of bipolar disorder exhibit seasonal 
variation? A multisite prospective investigation. Bipolar Disorders, 13(7-8), 687-695. doi:10.1111/j.1399-5618.2011.00959.x.

Natale, V., \& Cicogna, P. (2002). Morningness-eveningness: is it really a continuum? Personality \& Individual Differences, 32(5), 809-816.

Nathan, P. J., Burrows, G. D., \& Norman, T. R. (1999). Melatonin sensitivity to dim white light in affective disorders. Neuropsychopharmacology, 21(3), 408-413. doi:10.1016/s0893-133x(99)00018-4.

Nurnberger, J. I., Adkins, S., Lahiri, D. K., Mayeda, A., Hu, K., Lewy, A. J., et al. (2000). Melatonin suppression by light in euthymic bipolar and unipolar patients. Archives of General Psychiatry, 57(6), 572-579.

Ottoni, G. L., Lorenzi, T. M., \& Lara, D. R. (2011). Association of temperament with subjective sleep patterns. Journal of Affective Disorders, 128(1-2), 120-127. doi:10.1016/j.jad.2010.06.014.

Oyane, N. M. F., Bjelland, I., Pallesen, S., Holsten, F., \& Bjorvatn, B. (2008). Seasonality is associated with anxiety and depression: the Hordaland health study. Journal of Affective Disorders, 105(1-3), 147-155. doi:10.1016/j.jad.2007.05.002.

Parslow, R. A., Jorm, A. F., Butterworth, P., Jacomb, P. A., \& Rodgers, B. (2004). An examination of seasonality experienced by Australians living in a continental temperate climate zone. Journal of Affective Disorders, 80(2-3), 181-190. doi:10.1016/s0165-0327(03)00113-7.

Prociow, P. A., \& Crowe, J. A. (2010). Towards personalised ambient monitoring of mental health via mobile technologies. Technology and Health Care, 18(4-5), 275-284. doi:10.3233/thc-2010-0590.

Reichart, C. G., Van Der Ende, J., Wals, M., Hillegers, M. H. J., Ormel, J., Nolen, W. A., et al. (2004). The use of the GBI in a population of adolescent offspring of parents with a bipolar disorder. Journal of Affective Disorders, 80(2-3), 263-267.

Roberts, R. D., \& Kyllonen, P. C. (1999). Morningness-eveningness and intelligence: early to bed, early to rise will likely make you anything but wise! Personality and Individual Differences, 27(6), 1123-1133. doi:10.1016/s0191-8869(99)00054-9.

Rosenthal, N. E., Bradt, G. H., \& Wehr, T. A. (1984). Seasonal Pattern Assessment Questionnaire. Bethesda: National Institute of Mental Health.

Savitz, J., van der Merwe, L., \& Ramesar, R. (2008). Hypomanic, cyclothymic and hostile personality traits in bipolar spectrum illness: a family-based study. Journal of Psychiatric Research, 42(11), 920-929.

Schaffer, A., Levitt, A. J., \& Boyle, M. (2003). Influence of season and latitude in a community sample of subjects with bipolar disorder. Canadian Journal of Psychiatry, 48(4), 277-280.
Schweitzer, I., Maguire, K., \& Ng, C. H. (2005). Should bipolar disorder be viewed as manic disorder? Implications for bipolar disorder. Bipolar Disorders, 7, 418-423.

Shand, A. J., Scott, N. W., Anderson, S. M., \& Eagles, J. M. (2011). The seasonality of bipolar affective disorder: comparison with a primary care sample using the Seasonal Pattern Assessment Questionnaire. Journal of Affective Disorders, 132(1-2), 289-292. doi:10.1016/j. jad.2011.02.015.

Shen, G. H. C., Sylvia, L. G., Alloy, L. B., Barrett, F., Kohner, M., Iacoviello, B., et al. (2008). Lifestyle regularity and cyclothymic symptomatology. Journal of Clinical Psychology, 64(4), $482-500$.

Simonsen, H., Shand, A. J., Scott, N. W., \& Eagles, J. M. (2011). Seasonal symptoms in bipolar and primary care patients. Journal of Affective Disorders, 132(1-2), 200-208. doi:10.1016/j.jad.2011. 02.018 .

Smith, C. S., Reilly, C., \& Midkiff, K. (1989). Evaluation of three circadian rhythm questionnaires with suggestions for an improved measure of morningness. Journal of Applied Psychology, 74(5), 728-738.

Souetre, E., Salvati, E., Wehr, T. A., Sack, D. A., Krebs, B., \& Darcourt, G. (1988). Twenty-four-hour profiles of body temperature and plasma TSH in bipolar patients during depression and during remission and in normal control subjects. American Journal of Psychiatry, 145(9), 1133-1137.

Suhail, K., \& Cochrane, R. (1998). Seasonal variations in hospital admissions for affective disorders by gender and ethnicity. Social Psychiatry and Psychiatric Epidemiology, 33(5), 211217.

Tonetti, L., Barbato, G., Fabbri, M., Adan, A., \& Natale, V. (2007). Mood seasonality: a cross-sectional study of subjects aged between 10 and 25 years. Journal of Affective Disorders, 97(1-3), 155-160.

Wood, J., Birmaher, B., Axelson, D., Ehmann, M., Kalas, C., Monk, K., et al. (2009). Replicable differences in preferred circadian phase between bipolar disorder patients and control individuals. Psychiatry Research, 166(2-3), 201-209. doi:10.1016/j.psychres. 2008.03.003.

Young, M. A., Blodgett, C., \& Reardon, A. (2003). Measuring seasonality: psychometric properties of the Seasonal Pattern Assessment Questionnaire and the Inventory for Seasonal Variation. Psychiatry Research, 117(1), 75-83. 